\title{
Espacios intergubernamentales para la discusión de políticas migratorias en Latinoamérica
}

William Mejía Ochoa*

\section{RESUMEN}

El presente artículo pasa revista a los espacios regionales y subregionales con que cuentan los Gobiernos latinoamericanos para discutir asuntos migratorios y definir acciones conjuntas al respecto, y, a partir de las declaraciones conjuntas que se han adoptado recientemente, muestra los temas principales que preocupan a esos Gobiernos y los desafíos a los que se enfrentan para ser coherentes con lo acordado

Palabras clave: Latinoamérica, política migratoria, organizaciones intergubernamentales.

\section{Intergovernmental spaces for the discussion of migratory policies in Latin America}

\begin{abstract}
This article reviews the regional and sub-regional spaces that are available to Latin American governments to discuss migratory issues and to define joint actions related to these. Based on recent joint declarations, it showcases the issues that are important to these governments and the challenges that they face in order to be coherent with what has been accorded.

Keywords: Latin America, migration policy, intergovernmental organizations.
\end{abstract}

\footnotetext{
* MA en Migraciones Internacionales. Investigador Grupo de Investigación en Movilidad Humana (UTP-UNADSUEJE-CEMHCO) Bogotá (Colombia).wmejia8a@yahoo.com

Recibido: 22 de febrero de 2015 / Modificado: 5 de abril de 2015 / Aceptado: 7 de mayo de 2015 Para citar este artículo

Mejía Ochoa, W. (2015). Espacios intergubernamentales para la discusión de políticas migratorias en Latinoamérica. OASIS, 22, 7-21. DOI: http://dx.doi.org/10.18601/16577558.n22.02
} 


\section{INTRODUCCIÓN}

El espíritu de asociación e integración que anima hoy a Latinoamérica y a sus vecinos, así como el incremento de la migración intrarregional y de la importancia de varios países de ella como territorios de tránsito, incluso de flujos extracontinentales, con los principales destinos localizados en la periferia de la región (Chile, Argentina, Brasil y México) o fuera de ella (Estados Unidos y Canadá), asociado a la existencia de grupos importantes de desplazados y refugiados, producto de conflictos propios (Colombia) y extrarregionales (Siria, por ejemplo), además del incremento de los apátridas en el mundo, han sacado a la luz, de una manera especial en los años recientes, el tema de la población en movimiento a través de la región.

Tal situación ha llevado a que los asuntos migratorios, particularmente internacionales, se hayan posicionado en los escenarios de discusión política intergubernamental de la región, que a menudo hacen acuerdos y emiten declaraciones al respecto, con una eficacia que está por medirse y cuyo análisis queda por fuera de los alcances de este documento.

Aunque, en general, se trata de instrumentos no vinculantes, esto es, que no son de obligatorio cumplimiento, son importantes en la medida que corresponden a lo que se ha dado en llamar "derecho blando" y están constituidos por: declaraciones de principios que reflejan una visión política y estrategia común; lineamientos programáticos o guías que permiten planificar o implementar actividades; interpretaciones de los estándares contenidos en tratados internacionales; y principios rec- tores que permiten esclarecer obligaciones internacionales (CEPAL 2013, p. 6).

$\mathrm{El}$ artículo consta de tres secciones: una primera donde se hace un inventario clasificado de esos escenarios institucionales, con alguna referencia a su origen, composición y órganos principales, así como a su relación misional con los temas migratorios y las instancias especializadas con que cuentan para la discusión de tales temas; en la segunda se presentan los desafíos prácticos que enfrentan en el corto y mediano plazo los gobiernos de la región, si quieren ser consecuentes en la atención de las problemáticas migratorias más reiteradas en los pronunciamientos oficiales de las instituciones reseñadas de las que hacen parte; finalmente, se cierra el documento con algunas reflexiones que pretenden concluir.

\section{ESCENARIOS DE DISCUSIÓN DE LA TEMÁTICA MIGRATORIA EN AMÉRICA LATINA}

A continuación se reseñan los principales espacios intergubernamentales que constituyen escenarios para la discusión migratoria en Latinoamérica, los cuales tienen alcances geográficos y políticos distintos. Se empieza por los que tienen como objetivo estratégico la integración de sus afiliados, o de algunas de sus funciones en unidades políticas mayores, conformando bloques, para seguir con los que tienen propósitos centrados en el diálogo y la cooperación, y terminar con los creados ad hoc para el tratamiento de asuntos migratorios. Todos los reseńados corresponden a instituciones permanentes, a excepción del proceso Cartagena +30 , que es ya parte del pasado, pero que se incluyó por la importancia que 
tuvo en su cercano momento y por representar, a nuestro modo de ver, un ejemplo de mejor práctica para el tratamiento conjunto de asuntos migratorios y la definición de orientaciones para la implementación de políticas al respecto.

\section{BLOQUES SUBREGIONALES DE INTEGRACIÓN}

\section{Mercado Común del Sur (MERcosUR)}

El Mercosur se empezó a gestar en 1985, con un acuerdo de integración bilateral entre Brasil y Argentina, pero su creación propiamente dicha se produjo mediante el Tratado de Asunción, firmado en marzo de 1991. El MERCosur está integrado por Argentina, Brasil, Paraguay, Uruguay, Venezuela y Bolivia, y tiene como Estados asociados a Chile, Perú, Ecuador, Colombia, Guyana y Surinam.

El Mercosur cuenta con tres órganos cuyos acuerdos tienen carácter vinculante: el Consejo del Mercado Común (CMC), su órgano supremo, que emite Decisiones; el Grupo Mercado Común (GMC), que emite Resoluciones; y la Comisión de Comercio del Mercosur (ссм), que emite Directivas.

En relación con el tema migratorio, MERCosur ha tenido diferentes espacios, todos en el marco de la Reunión de Ministros del Interior: Comisión de Asuntos Migratorios, Subcomisión de Seguimiento y Control del Área Migratoria, Grupo Especializado de Trabajo Migratorio, Comisión Técnica y Foro Especializado Migratorio (FEM), el más importante de todos.

$1 \quad$ Véase al respecto http://www.mercosur.int/
El Foro Especializado Migratorio del MERCOSUR y Estados asociados fue creado en la XIV Reunión de Ministros del Interior del MERCOSUR, en 2003, realizada en la ciudad de Montevideo, y tuvo su primera reunión en 2004. El FEM tiene entre sus funciones el estudio del impacto de las migraciones en la región y fuera de ella, y el análisis y desarrollo de proyectos de normas o acuerdos en materia migratoria para los países del bloque (Mercosur, 2015).

Algunas medidas internas de MERCosur asociadas a lo migratorio han tenido impacto en el resto de la región, particularmente en Suramérica, y han logrado la vinculación de otros países, como: el Acuerdo sobre Residencia para Nacionales de los Estados Partes; el Protocolo de Integración Educativa y Reconocimiento de Certificados, Títulos y Estudios de Nivel Primario y Medio No Técnico; y el Acuerdo sobre la Creación e Implementación de un Sistema de Acreditación de Carreras Universitarias para el Reconocimiento Regional de la Calidad Académica de las respectivas Titulaciones ${ }^{1}$.

\section{Comunidad Andina (CAN)}

En 1969, con la firma del Acuerdo de Cartagena, inició un proceso de integración subregional, que también se conoció como Pacto Andino o Grupo Andino y que hoy se conoce como Comunidad Andina, compuesta por Bolivia, Colombia, Ecuador y Perú, cuyo Consejo Andino de Ministros expide Decisiones que tienen carácter vinculante. 
La CAN se planteó la movilidad de sus ciudadanos y avanzó significativamente al respecto, con logros adoptados mediante Decisiones, como: la posibilidad de viajar al interior de la subregión, en calidad de turista, con apenas el documento de identidad nacional; la existencia de garantías comunes y portabilidad de la seguridad social para los trabajadores migrantes; la existencia de un pasaporte andino y de la Tarjeta Andina de Migración (TAM); el Instrumento Andino de Migración Laboral (Decisión 545), que ofrece a los trabajadores andinos el mismo trato que a los nacionales en el territorio comunitario; y mecanismos para las equivalencias de los estudios de educación media. Para los logros anteriores fueron clave el Comité Andino de Autoridades de Migración (CAAM) y su subcomité dedicado a las migraciones laborales (CAAM Laboral).

No obstante, el tema migratorio parece haber perdido importancia dentro de la dinámica de la CAN, y las últimas actividades al respecto corresponden al 2013, cuando, durante el primer semestre, se reglamentó el Mecanismo Andino de Cooperación en materia de Asistencia y Protección Consular y Asuntos Migratorios, y se realizó el Iv Foro Andino de Migraciones. Cabe comentar que la Propuesta de Decisión sobre "El Plan Andino de Desarrollo Humano para las Migraciones", elaborada en abril de 2012, sigue en espera de aprobación por parte del Consejo Andino de Ministros de Relaciones Exteriores.

\section{Sistema de Integración Centroamericana (SICA)}

El Sistema de Integración Centroamericana (sICA) se constituyó en diciembre de 1991, mediante el Protocolo de Tegucigalpa, que modificó la Carta de la Organización de Estados Centroamericanos (oDECA), surgida en 1951. En la Cumbre Extraordinaria de Jefes de Estado y de Gobierno, en julio de 2010, se reafirmaron los compromisos integracionistas y se hizo un relanzamiento del sica en el que se priorizaron los siguientes temas: seguridad democrática, prevención y mitigación de los desastres naturales y de los efectos del cambio climático, integración social, integración económica y fortalecimiento de la institucionalidad regional. Las resoluciones de su Comité Ejecutivo tienen carácter vinculante.

El sicA está integrado por Costa Rica, El Salvador, Guatemala, Honduras, Nicaragua, Panamá, Belice y República Dominicana. Adicionalmente, cuenta con un grupo de países Observadores Regionales y Extrarregionales: México, Chile, Brasil, Argentina, Perú, Estados Unidos, Ecuador, Uruguay, Colombia, China (Taiwán), España, Alemania, Italia, Japón, Australia, Corea del Sur, Francia, la Santa Sede, Reino Unido, Unión Europea, Nueva Zelandia, Marruecos y Qatar. Haití se encuentra en proceso de incorporación bajo la categoría de Observador Regional.

Como instancia especializada en materia migratoria, el sicA cuenta con la Comisión Centroamericana de Directores de Migración (осAм), vinculada a la Secretaría de Integración Económica Centroamericana (sIECA). Como mayor logro en el tema, el sica muestra el acuerdo de libre movilidad y sin pasaporte entre Guatemala, El Salvador, Honduras y Nicaragua, aunque deben considerarse otros esfuerzos, como el realizado a través de la Coordinación Educativa y Cultural Centroame- 
ricana para la homologación de los sistemas educativos ${ }^{2}$.

\section{Unión de Naciones Suramericanas (UNASUR)}

Para integrar procesos regionales desarrollados por el mercosur y la CAN, en la Reunión de Presidentes de América del Sur, que se realizó en diciembre de 2004, se creó la Comunidad Suramericana de Naciones (CSN), que cambió su nombre en abril de 2007 por el de Unión de Naciones Suramericanas (UNASUR), cuyo Tratado Constitutivo se aprobó en mayo de 2008, y entró en vigencia el 11 de marzo de $2011^{[3]}$.

Sus creadores fueron Argentina, Bolivia, Brasil, Colombia, Chile, Ecuador, Guyana, Paraguay, Perú, Surinam, Uruguay y Venezuela, que definieron como objetivo principal "construir un espacio de integración en lo cultural, económico, social y político, respetando la realidad de cada nación". Sus órganos principales son: el Consejo de Jefas y Jefes de Estado y de Gobierno, el Consejo de Ministras y Ministros de Relaciones Exteriores; y el Consejo de Delegadas y Delegados (UNAsur, 2014), cuyos acuerdos no tienen carácter vinculante. También cuenta con una Secretaría General, una Presidencia Pro-Témpore y doce Consejos Ministeriales.

Dentro de los objetivos específicos definidos en el Tratado Constitutivo, figuran dos directamente relacionados con lo migratorio:
La consolidación de una identidad suramericana a través del reconocimiento progresivo de derechos a los nacionales de un Estado Miembro residentes en cualquiera de los otros Estados Miembros, con el fin de alcanzar una ciudadanía suramericana.

La cooperación en materia de migración, con un enfoque integral, bajo el respeto irrestricto de los derechos humanos y laborales para la regularización migratoria y la armonización de políticas (UNASUR, 2014).

\section{ESPACIOS DE DIÁLOGO Y COOPERACIÓN}

\section{Organización de los Estados Americanos (OEA)}

Con antecedentes que se remontan a finales del siglo xIx, en 1948 se suscribió la Carta constitutiva de la Organización de los Estados Americanos (OEA), que entró en vigencia en diciembre de 1951 y que plantea como objetivo para sus miembros "un orden de paz y de justicia, fomentar su solidaridad, robustecer su colaboración y defender su soberanía, su integridad territorial y su independencia”.

La OEA reúne a los 35 Estados independientes de las Américas, y constituye el mayor foro gubernamental de la región, donde el diálogo político - quizás su función más importante- se lleva a cabo en diferentes instancias, como el Consejo Permanente, las reuniones regionales de ministros, la Asamblea General y las Cumbres de las Américas, instancias en las cuales el tema migratorio ha sido en algún momento objeto de consideración.

2 Véase al respecto http://www.sica.int/

3 Véase al respecto http://www.unasursg.org/ 
Como foro especializado y principal para la discusión migratoria, la oEA cuenta con la Comisión de Asuntos Migratorios (CAM), que inició labores en julio de 2012 en condición de comisión permanente de su Consejo Interamericano para el Desarrollo Integral (CIDI). La CAM reemplazó a la Comisión Especial de Asuntos Migratorios (CEAM), que funcionaba, adscrita al Consejo Permanente, desde abril de 2008.

De otro lado, la oea tiene el Programa Inter-Americano para la Promoción y Protección de los Derechos Humanos de los Migrantes, incluido el llamado a la firma, ratificación o adhesión a los instrumentos universales e interamericanos de derechos humanos y derechos de los migrantes, que tienen carácter vinculante, así como a la promoción de políticas públicas y legislación eficaces y coherentes con esos instrumentos. Tal programa dispone de las Sesiones Especiales de la Comisión de Asuntos Jurídicos y Políticos (CAJP) sobre los Derechos Humanos de los Migrantes, que constituyen un foro donde los Estados miembros, entre otras cosas, conocen mejores prácticas al respecto ${ }^{4}$.

\section{Conferencia Iberoamericana}

La Conferencia Iberoamericana de Jefes de Estado y de Gobierno cuenta con la participación de los Estados soberanos de América y Europa de lengua española y portuguesa, y surgió como producto de la primera Cumbre que reunió a tales jefes en 1991.

La Conferencia tiene en la Cumbre de Jefes de Estado y de Gobierno que se realiza anualmente, y en la Reunión de Ministros de Relaciones Exteriores, sus más altas instancias políticas. Por su parte, las Reuniones Ministeriales Sectoriales, también anuales, reúnen a los ministros y altos responsables iberoamericanos de todas las áreas.

Dentro de su estructura, la Conferencia cuenta con la Organización Iberoamericana de Seguridad Social, entre cuyos logros se cuenta el Convenio Multilateral Iberoamericano de Seguridad Social, que busca la coordinación de legislaciones nacionales en materia de pensiones, como garantía de la seguridad económica en la vejez, la incapacidad o la muerte, lo que beneficia directamente a los trabajadores migrantes, en la medida que les permite la portabilidad de tales pensiones 5 .

Otros de sus organismos no directamente relacionados con el tema migratorio pueden ser circunstancialmente ejecutores de propuestas con capacidad de incidir en las políticas migratorias regionales. Tal es el caso de la Secretaría General Iberoamericana (sEgiB) y de la Organización de Estados Iberoamericanos para la Educación, la Ciencia y la Cultura (OEI), a quienes la última Cumbre encomendó la promoción de una "Alianza para la Movilidad Académica”, para la movilidad de estudiantes, profesores e investigadores, con el objeto de

\footnotetext{
Véase al respecto http://www.oas.org/es/temas/migracion.asp

Véase al respecto http://www.oiss.org/
} 
prevenir la ocurrencia y mitigar el "impacto negativo de la fuga de cerebros"; asimismo, encargó a la SEGIB el estudio de la viabilidad de un Convenio Marco para impulsar dentro del espacio iberoamericano la facilitación de prácticas y pasantías de estudios de duración limitada, la movilidad intraempresarial de directivos y trabajadores, la movilidad de profesionales titulados e investigadores y la movilidad de inversores y emprendedores (Cumbre Iberoamericana, 2014).

\section{Comunidad de Estados Latinoamericanos y del Caribe (CELAC)}

La CELAC es un mecanismo intergubernamental representativo de diálogo y concertación política, que reúne de manera permanente a los 33 países de América Latina y el Caribe, y actúa sobre la base del consenso. Se constituyó oficialmente mediante la Declaración de Caracas en la Cumbre de Latinoamérica y el Caribe (Calc), en diciembre de 2011. Dentro de sus órganos, el principal es la Cumbre de Jefes de Estado y de Gobierno, que sesiona durante dos días una vez al año y culmina con la adopción de la Declaración Política de la Cumbre, el Plan de Acción y las Declaraciones Especiales, que carecen de carácter vinculante. Otros órganos son: la Reunión de Ministros de Relaciones Exteriores, la Presidencia Pro Témpore y las Reuniones Sectoriales, entre las que están las migratorias (CELAC, 2015).

Una de las atribuciones de la CELAC es la interlocución con otros países y grupos regionales, entre la que se destaca el diálogo con la Unión Europea, que cuenta con el Diálogo Estructurado sobre Migración, acordado en la v Cumbre ALC-UE en 2008 y que tuvo su primera Reunión UE-CELAC de Alto Nivel sobre Migración en 2009, después de la cual se han realizado otras ocho, la última de las cuales se llevó a cabo en noviembre de 2014. Las temáticas de las nueve reuniones han sido, en su orden, las siguientes: sinergias entre migración y desarrollo; educación, salud y migración; familias, grupos vulnerables y migración; migración irregular; migración regular; migración y crecimiento económico; trata de personas y tráfico de migrantes; políticas para protección e integración de migrantes y lucha contra todas las formas de discriminación, racismo y xenofobia.

El principal proyecto en este campo, financiado por la Unión Europea, es "Fortalecimiento del diálogo y de la cooperación entre la Unión Europea (UE) y América Latina y el Caribe (ALC) para el establecimiento de modelos de gestión sobre migración y políticas de desarrollo", con el objetivo de contribuir al proceso de cooperación entre la UE y la CELAC, $y$ al fortalecimiento de las capacidades regionales y nacionales de los países de esta, a fin de apoyar el intercambio permanente de información y buenas prácticas tanto entre ellos, como entre la CELAC y la Ue (OIM-FIIAPP, 2011).

\section{ESPACIOS ESPECIALIZADOS}

\section{Conferencia Suramericana sobre Migraciones} (CSM)

La Conferencia Suramericana sobre Migraciones (CSM) surgió a partir de la iniciativa de Gobiernos suramericanos durante el Encuentro Sudamericano sobre Migraciones, Integra- 
ción y Desarrollo, realizado en Lima en 1999. Constituye una instancia de decisión política orientada a generar y coordinar iniciativas y programas dirigidos a promover y desarrollar políticas sobre las migraciones internacionales y su relación con el desarrollo y la integración regional. La primera CSM se llevó a cabo en 2000, en Buenos Aires, y a partir de allí se ha realizado anualmente una, hasta la XIv, reunida en Lima en octubre de 2014.

Actualmente, está integrada por Argentina, Bolivia, Brasil, Chile, Colombia, Ecuador, Guyana, Paraguay, Perú, Surinam, Uruguay y Venezuela. Como observadores incluye a organismos internacionales, representantes de la sociedad civil y de gobiernos específicos, que pueden participar en las actividades de la Conferencia, pero no en la elaboración de los documentos que contengan recomendaciones o compromisos.

En la Declaración de principios migratorios y lineamientos generales de la Conferencia Sudamericana de Migraciones, aprobada en sus reuniones de 2009 y 2010, se definió como un "espacio de diálogo, intercambio y construcción colectiva, que los países suramericanos han generado sobre el proceso migratorio en la región" (CSM, 2010)

En la x csm se aprobó el Plan Sudamericano de Desarrollo Humano de las Migraciones, que "tiene como objetivo principal recoger los principios y derechos que se han consensuado en el desarrollo de las diferentes conferencias; plantear los lineamientos estratégicos que surgen de dichos principios y derechos; y proponer programas de acción acordes a los lineamientos estratégicos planteados" (CSM, 2010a).
La agenda de la última CSM contempló los siguientes ejes temáticos: seguimiento al Plan Suramericano de Desarrollo Humano de las Migraciones; las migraciones y la integración suramericana; fortalecimiento de la gestión migratoria; y la proyección internacional de la región suramericana en materia migratoria.

\section{Conferencia Regional sobre Migración (CMR)}

La Conferencia Regional sobre Migración (CMR) se gestó en la Cumbre de Presidentes Tuxtla II en febrero de 1996, y su primera reunión se llevó a cabo el mes siguiente en Puebla (México), por lo que también se le conoce como "Proceso de Puebla". Es un foro regional intergubernamental, donde se intercambia información, se comparten experiencias y mejores prácticas, y se realizan consultas tendientes a fomentar la cooperación en materia migratoria. Su Plan de Acción se centra en: políticas migratorias y gestión, derechos humanos y migración y desarrollo. Las decisiones de la CRM, a cargo de la Reunión Anual de los Viceministros (Gobierno y Relaciones Exteriores), no tienen carácter vinculante pero constituyen "un marco de cooperación" (CRM, 2013).

Los países miembros de la CMR son Belice, Canadá, Costa Rica, El Salvador, Estados Unidos, Guatemala, Honduras, México, Nicaragua, Panamá y República Dominicana. Como observadores se encuentra Argentina, Colombia, Ecuador, Jamaica y Perú, además de un conjunto de organizaciones internacionales: Comisión Interamericana de Derechos Humanos (CIDH), Organización Internacional para las Migraciones (OIM), Comisión Económica para América Latina y el Caribe de las 
Naciones Unidas / Centro Latinoamericano y Caribeño de Demografía (CEPAL/CELAde), Oficina del Alto Comisionado de las Naciones Unidas para los Refugiados (ACNUR), Relatoría Especial sobre los Derechos Humanos de los Migrantes de la Organización de las Naciones Unidas, Sistema de la Integración Centroamericana (SICA), Fondo de Población de las $\mathrm{Na}$ ciones Unidas (Unfra), Secretaría General de la Conferencia Iberoamericana (SEGIB) y Comité Internacional de la Cruz Roja (CICR). Se invita a organizaciones de la sociedad civil (OsC) a participar en los seminarios, talleres y otros eventos de la CRM, cuando corresponde.

\section{$30^{\circ}$ aniversario de la Declaración}

de Cartagena sobre los Refugiados

Durante 2014, se realizó el proceso conmemorativo de los treinta años de la Declaración de Cartagena sobre Refugiados (1984), que "amplió la definición de refugiado para América Latina y propuso nuevos enfoques para las necesidades humanitarias de las personas refugiadas y desplazadas con un espíritu de solidaridad y cooperación".

Tal conmemoración, conocida como Cartagena+30, incluyó cuatro reuniones preparatorias subregionales, que contaron con la participación de gobiernos, organismos internacionales y regionales, defensorías del pueblo, sociedad civil y academia, y terminó el 2 y 3 de diciembre de 2014, con un conjunto de actos que incluyeron una Reunión Ministerial en la que 28 países y tres territorios de América
Latina y el Caribe aprobaron por aclamación la Declaración y el Plan de Acción de Brasil, "acordando trabajar juntos para mantener los estándares de protección más altos a nivel internacional y regional, implementar soluciones innovadoras para las personas refugiadas y desplazadas, y ponerle fin a la difícil situación que enfrentan las personas apátridas en la región"“6.

\section{PRINCIPALES DESAFÍOS ACTUALES EN LA DETERMINACIÓN DE POLÍTICAS Y ACCIONES MIGRATORIAS EN AMÉRICA LATINA}

La revisión de los acuerdos políticos recientes en torno a asuntos migratorios logrados al interior de las instituciones reseñadas, que además de representar posiciones conjuntas marcan línea, que no siempre se sigue, para la definición de políticas y acciones por parte de cada uno de los gobiernos implicados, permite identificar las preocupaciones regionales actuales al respecto, y las definiciones de política migratoria y acciones relacionadas que se esperarían en los niveles nacionales en el futuro cercano, sobre el supuesto de coherencia de sus gobiernos con lo acordado regionalmente.

A continuación, se presentan los principales desafíos que implican lo que parecen ser las mayores o más comunes de esas preocupaciones, tomando como referencia los acuerdos consignados en declaraciones oficiales de las entidades en cuestión, realizadas durante 2014 y enero de 2015.

6 Véase al respecto http://www.acnur.org/cartagena30/ 


\section{HACER EFECTIVOS LOS DERECHOS DE LOS MIGRANTES Y DE OTRAS POBLACIONES EN MOVIMIENTO DENTRO DE LA REGIÓN}

A este asunto se refirió en enero de 2014 la II Cumbre de la Comunidad de Estados Latinoamericanos y Caribeńos (CELAC), y sus Estados se comprometieron a formular nuevas políticas y a fortalecer las existentes, con el fin de reconocer a los migrantes como sujetos de derecho y facilitar la regularización migratoria (CELAC, 2014). En los mismos términos lo hizo la XIV Conferencia Suramericana sobre Migraciones (CSM), y fue más allá, al considerar la inclusión social de los migrantes como una de las bases del proceso de integración regional, para lo cual,

se debe promover su acceso, en igualdad de condiciones con los nacionales, a todos los derechos, entre otros, al trabajo, a la seguridad social, a la salud, a la justicia, a la vivienda, a la educación, a la participación social y política, así como a la cultura en las sociedades de acogida (CSM, 2014).

Tres grupos llamaron la atención de los países de la región en cuanto a la urgencia de atención en pro de sus derechos: niños y adolescentes migrantes no acompańados, particularmente a Estados Unidos; desplazados y refugiados; y apátridas. Al primero aludió la CELAC en reunión posterior a la II Cumbre (CELAC, 2014b), que definió trabajar en un protocolo regional para su atención, como lo mencionaron tam- bién MERCOSUR (2014a y 2014b), la Organización de Estados Americanos (OEA) (2014) y la Conferencia Regional sobre Migración (CRM), que lo definió como "un reto que requiere una respuesta regional en materia de prevención, protección, retorno y reintegración" (CRM, 2014a); para priorizar acciones al respecto se adoptó una Declaración Extraordinaria (CRM, 2014b).

La III Cumbre de la CELAC fue reiterativa en estas cuestiones y destacó que la garantía del pleno respeto de todos los derechos humanos de las personas migrantes, que cabe a los Estados de origen, tránsito y destino, es “independiente de su estatus migratorio, incluyendo la migración de niños, niñas y adolescentes, acompańados y no acompańados y su interés superior, evitando la exacerbación de las vulnerabilidades" (CELAC, 2015b).

Sobre los desplazados, refugiados y apátridas, los países de América Latina y el Caribe, reunidos para conmemorar el trigésimo aniversario de la Declaración de Cartagena sobre los Refugiados, asumieron compromisos importantes en una declaración ${ }^{7}$ (Cartagena+30 2014) y un plan de acción, cuyos programas tienen nombres que indican sus objetivos: Asilo de Calidad; Fronteras solidarias y seguras; Repatriación voluntaria; Integración local; Reasentamiento solidario; Movilidad Laboral; Observatorio de los derechos humanos para el desplazamiento; Prevención; Tránsito digno y seguro; Erradicación de la condición de apátrida (Cartagena+30, 2014b). El compromiso

\footnotetext{
7 Aprobada por aclamación en la clausura de la Reunión Ministerial, por los representantes de 28 países y tres territorios.
} 
con ambos documentos fue recalcado por los presidentes de los Estados partes y Estados asociados del MERCOSUR (2014b).

Tales compromisos implican, entre otras acciones y según lo acordado en varios de los documentos citados y en otros, una campaña regional de adhesión o ratificación por parte de los Estados que aún no lo han hecho, así como el desarrollo progresivo de la interpretación de, por lo menos, estos instrumentos: la Convención Internacional sobre la Protección de los Derechos de Todos los Trabajadores Migratorios y de sus Familiares de 1990; la Convención sobre los Derechos del Niño de 1989; la Convención sobre el Estatuto de los Refugiados de 1951 y su Protocolo de 1967; la Convención sobre el Estatuto de los Apátridas de 1954, la Convención para Reducir los Casos de Apatridia de 1961; la Convención de las Naciones Unidas contra la Delincuencia Organizada Transnacional de 2000 y sus Protocolos para Prevenir, Reprimir y Sancionar la Trata de Personas, Especialmente Mujeres y Niños, y contra el Tráfico Ilícito de Migrantes por Tierra, Mar y Aire (Protocolos de Palermo).

\section{VINCULAR LAS POLÍTICAS MIGRATORIAS CON OTRAS POLÍTICAS SOCIALES Y ECONÓMICAS, Y CON LOS PROPÓSITOS DEL DESARROLLO}

Los gobiernos de la región deberán avanzar próximamente en la vinculación de las políticas migratorias con otras políticas sociales y eco- nómicas, e integrarlas dentro de sus planes de desarrollo, como también se han comprometido, de manera general, a acoger la Declaración del Diálogo de Alto Nivel sobre la Migración Internacional y el Desarrollo ${ }^{8}$ (CELAC, 2014) y promover la incorporación de la migración en la Agenda de Desarrollo Post $2015^{\circ}$ (csm, 2014) y, particularmente, en casos como los de las personas refugiadas, desplazadas y apátridas (Cartagena+30 2014 y 2014a) o de niños, niñas y adolescentes migrantes no acompańados (CMR, 2014b).

En su III Cumbre, la CELAC ha vuelto sobre el tema, al declarar que "No puede haber desarrollo sostenible sin la inclusión de los grupos en situación de vulnerabilidad, tales como los pueblos indígenas y otros pueblos tribales, afrodescendientes, mujeres, personas adultas mayores, personas con discapacidad, migrantes, niños, niñas y adolescentes", y ratificar el vínculo entre migración internacional y desarrollo, referido también a los países de destino (Celac, 2015b). La misma Cumbre, en su Plan de Acción, estableció "continuar la coordinación CELAC de cara al Diálogo Estructurado sobre Migraciones CELAC-UE, en cuyo marco se procurará fortalecer el vínculo positivo entre migración, desarrollo y derechos humanos" (CELAC 2015c).

Si se considera la importancia del trabajo en los asuntos migratorios, hay un desafío para avanzar de manera especial en la asociación de las políticas sobre ambos temas, particularmen-

\footnotetext{
8 Véase http://www.un.org/es/comun/docs/?symbol=A/68/L.5

9 Véase file:///D:/PROYECTOS\%2OEN\%2OCURSO/OIM\%2OMMP\%2O2O I 5/NACIONES\%2OUNIDAS\%2OPOST\%2O2OI $5 \% 20$ -\%2OSPANISH\%20-\%2OJULY\%2008.pdf
} 
te en la subregión centroamericana, donde un memorando de entendimiento firmado con la Organización Internacional del Trabajo (оIт) busca, entre otras cosas, incrementar la incidencia de los ministerios de trabajo, organizaciones representativas de empleadores y de trabajadores, en los espacios de discusión y construcción de las políticas migratorias laborales; vincular los programas de servicio público de empleo en la atención de las personas trabajadoras migrantes, en especial de mujeres y jóvenes, y formar personal en estos programas en el área de la movilidad laboral; abrir espacios de discusión sobre la seguridad social de las personas trabajadoras migrantes y su portabilidad; diseñar mecanismos efectivos de protección social para los trabajadores temporales (OIT-SICA, 2014).

\section{AVANZAR EN COOPERACIÓN INTRARREGIONAL Y SUR-SUR EN GENERAL}

El tercer gran desafío en materia de política migratoria para América Latina, será empezar a superar los ámbitos subregionales de cooperación, particularmente los de los acuerdos de integración de mayor antigüedad (sICA, CAN, MERCOSUR), y concretar en los espacios más amplios, como UNASUR y CELAC, y fuera de la región, la cooperación sur-sur en el tema que se ha declarado en varios documentos durante 2014, como la declaración de la reunión conmemorativa del el trigésimo aniversario de la Declaración de Cartagena sobre los Refugiados, en la que se hizo "un llamado colectivo para profundizar los niveles de articulación, complementariedad, cooperación y convergencia entre los mecanismos regionales y subregionales de integración, incluso en los temas relacionados con la migración, las personas refugiadas, desplazadas y apátridas”, y se propuso "avanzar hacia una progresiva armonización de políticas públicas, normas y procedimientos mediante el intercambio de buenas prácticas en materia de protección de las personas refugiadas, desplazadas y apátridas", teniendo en cuenta, entre otras cosas, un enfoque integral y diferencial de edad, género y diversidad (Cartagena+30, 2014a y 2014b).

En términos de la Conferencia Suramericana de Migraciones, es necesario mantener un enfoque integral para el tratamiento de los desafíos de la migración internacional, a partir del concepto de gobernanza, tomando en cuenta que los esfuerzos deben articular el trabajo conjunto de los Estados, con los aportes de los organismos internacionales y demás actores vinculados a la temática, y debe destacarse la relevancia de la cooperación consular y horizontal como herramientas que contribuyen al tratamiento integral de las migraciones, a fin de profundizar la cooperación sur-sur (CSM, 2014).

\section{CONCLUSIONES}

Latinoamérica cuenta con un importante número de instancias intergubernamentales, algunas con espacios para la participación de la sociedad civil, donde se desarrolla diálogo político en torno a los asuntos migratorios que competen a la región y se posibilita el logro de consensos.

Tales espacios tienen diversos y cambiantes alcances geográficos y de incidencia, relacionados con muchos aspectos, específicamente 
geopolíticos, entre los cuales podría estar la búsqueda de autonomía regional, en particular frente a sus vecinos del norte, en especial Estados Unidos. Esta situación podría explicar un aparente mayor activismo reciente en el tema por parte de instancias como el CELAC.

Un análisis más puntual deberá considerar las implicaciones reales de las declaraciones y compararlas con el impacto práctico de situaciones de hecho, a veces poco publicitadas, como la ampliación, por adhesión, del espacio del convenio de residencia de MERCOsur.

Las declaraciones consensuadas durante el último año (anterior a la producción de este documento) en las instancias consideradas, indican que los mayores desafíos en el futuro cercano que los gobiernos de la región enfrentan para ser consecuentes con lo acordado son: hacer efectivos en sus territorios los derechos de los migrantes y de otra población en movimiento, y vincular efectivamente las políticas migratorias con otras políticas sociales y económicas, y con sus propósitos de desarrollo.

\section{REFERENCIAS}

Cartagena+30 (2014a). Declaración de Brasil. Un marco de cooperación y solidaridad regional para fortalecer la protección internacional de las personas refugiadas, desplazadas y apátridas en América Latina y el Caribe. Recuperado de http://www. acnur.org/t3/fileadmin/scripts/doc.php?file=t3/ fileadmin/Documentos/BDL/2014/9867

Cartagena+30 (2014b). Plan de Acción de Brasil. Una hoja de ruta común para fortalecer la protección y promover soluciones sostenibles para las personas refugiadas, desplazadas y apátridas en América Latina y el Caribe dentro de un marco de coope- ración y solidaridad. Recuperado de http://www. acnur.org/t3/fileadmin/scripts/doc.php?file=t3/ fileadmin/Documentos/BDL/2014/9867

CElaC (2015a). ¿Qué es Celac? Cuadernos de Celac 1. Recuperado de http://www.celac2015.go.cr/wpcontent/uploads/2014/pdf/CELAC \%20Que\%20 es.pptx

CELAC (2015b). Declaración política de Belén III. Cumbre de jefas y jefes de Estado y de gobierno de la Comunidad de Estados Latinoamericanos y Caribeńos (CELAC). Recuperado de http://www. celac2015.go.cr/wp-content/uploads/20 I 5/o I / DECLARACION-POLITICA-DE-BELEN-20 I 5-ES-5.pdf CELAC (2015c). Plan de Acción de la CELAC, 2015. Recuperado de http://www.celac2015.go.cr/plan-deaccion-de-la-celac-2015/

CELAC (2014a). Declaración especial sobre la regularización migratoria como un mecanismo para lograr el ejercicio pleno de los derechos de las personas migrantes y sus familiares de los países miembros de la CELAC y el fortalecimiento de la integración regional. II Cumbre de la Comunidad de Estados Latinoamericanos y Caribeños. Recuperado de http://csm-osumi.org/Archivos/ Eventos/doc_3_15_regularizacion_migratoria_espanol.pdf

CELAC (2014b). Declaraciones finales de la III Reunión sobre migraciones de la CELAC. Recuperado de http://www.livestream.com/cancilleriaecua/ video?clipId=pla_6d6cbe20-5002-41ee-be1b8e76648df303

CEPAl (20I3). Tipología DE INSTRUMENTOS INTERNACionales. Preparado para el grupo de trabajo SOBRE DERECHOS DE ACCESO E INSTRUMENTO regional. Tercera Reunión de los Puntos FoCales Designados por los Gobiernos de los Países Signatarios de la Declaración sobre LA APLICACIÓN DEL PRINCIPIO IO DE LA DECLA- 
Ración de Río sobre el Medio Ambiente y el Desarrollo en América Latina y el Caribe. RECUPERADO DE HTTP:/WWW.CEPAL.ORG/RIO2O/ NOTICIAS/NOTICIAS/I/5079I/20I3-86I_PRIO_ TiPologia_InSTRUMENTOS.PDF

CRM (2014a). Declaración por una región libre de trata de personas, xIx Conferencia Regional sobre Migración, junio 27, 2014. http://www.acnur.org/ t3/fileadmin/scripts/doc.php?file=t3/fileadmin/ Documentos/BDL/2014/9605

CRM (2014b). Declaración Extraordinaria de Managua, XIx Conferencia Regional sobre Migración. Recuperado de http://www.iom.int/files/live/ sites/iom/files/What-We-Do/docs/Declaracionextraordinaria-Final-EsP.pdf

CRM (2013). La Conferencia Regional sobre Migración (CRM) o Proceso Puebla: antecedentes, miembros y observadores, el proceso, plan de acción, resultados de la CRM, principios. Recuperado de http://www.crmsv.org/Acerca_de/Brochure $\% 20$ de\%20la\%20CRM\%20-\%20Espa\%F1ol\%20 -\%202013\%20final.pdf

CSM (2010a). Declaración de principios migratorios y lineamientos generales de la Conferencia Sudamericana de Migraciones. Recuperado de http://csm-osumi.org/Archivos/Doccsm/2\%20 Declaraci\%C3\%B3n\%20principios\%20ES.pdf CSM (2010b). Plan sudamericano de desarrollo humano de las migraciones, contexto y perspectivas. Documento aprobado en la Décima Conferencia Sudamericana de Migraciones. Recuperado de https://www.iom.int/files/live/sites/iom/files/What-We-Do/docs/Plan-Sudamericano-deDesarrollo-Humano-de-las-Migraciones-PsDHM-2010.pdf

CSM (2014). Declaración de Lima. Migración e inclusión: un reto para la integración suramericana. XIV Conferencia Suramericana sobre Migraciones.
Recuperado de http://csm-osumi.org/Archivos/ Confcsm/Declaraci\%C3\%B3n\%20xiv\%20 Csm\%20Eng.pdf

Cumbre Iberoamericana (2014a). Declaración de Veracruz: "Educación, innovación y cultura en un mundo en transformación”. xxiv Cumbre Iberoamericana de Jefes de Estado y de Gobierno. Recuperado de http://www.segib.org/sites/default/ files/1.Declaracion\%20Veracruz\%20JEG-E.pdf

Cumbre Iberoamericana (2014b). Comunicado especial sobre los niños, niñas y adolescentes migrantes. xxIV Cumbre Iberoamericana de Jefes de Estado y de Gobierno. Recuperado de http://www.segib. org/sites/default/files/4.\%20Once\%20comUNICADOS\%2OESPECIALES.pdf

Mercosur (201 5). Foro Especializado Migratorio del mercosur y Estados Asociados. ReuNión de Ministros del Interior. Memoria InSTITUCiONAL. ReCUPERADO DE HTTP://www. MIGRACIONES.GOV.AR/FORO_MIGRATORIO/PDF/ MEMORIA_INSTITUCIONAL.PDF

MERCosur (2014a). Declaración especial de los Estados partes y Estados asociados del MERCOSUR sobre la situación de los niños, niñas y adolescentes centroamericanos migrantes retenidos en la frontera sur de los Estados Unidos de América. Recuperado de http://www.mercosur.int/t_generic.jsp ?contentid=6075\&site $=1 \&$ channel=secretaria\# mercosur (2014b). Comunicado conjunto de las presidentas y los presidentes de los Estados partes del mercosur y Estados asociados. XLVIL Reunión ordinaria del Consejo del Mercado Común (CMC). Recuperado de http://www.mercosur.int/ innovaportal/file/6166/1/comunicadoepyasociados-final.doc

OEA (2014). Declaración: Las niñas, niños y adolescentes centroamericanos migrantes no acompańados. Consejo Permanente de la Organización de Es-

OASIS, N²2 Julio-Diciembre 2015 - pp. 7-21 
tados Americanos. Recuperado de http://www. oas.org/es/centro_noticias/comunicado_prensa. asp?sCodigo $=\mathrm{D}-008 / 14$

OIM-FIIAPP (2011). Unión Europea - América Latina y el Caribe. Nota Informativa "Fortalecimiento del diálogo y de la cooperación entre la Unión Europea (UE) y América Latina y el Caribe (ALC) para el establecimiento de modelos de gestión sobre migración y políticas de desarrollo. Recuperado de http://www.migracion-ue-alc.eu/documents/keydocs/Es/Nota\%20informativa $\% 20$ _\%20proyecto\%20UE-ALC.pdf
OIT-SICA(2014). Memorando de Entendimiento entre el Sistema de la Integración Centroamericana (SICA), el Consejo de Ministros de Trabajo de Centroamérica y República Dominicana y la Organización Internacional del Trabajo (огт). Recuperado de http://www.ilo.org/wcmsp5/groups/public/--americas/---ro-lima/---sro-san_jose/documents/ legaldocument/wcms_327494.pdf

UNASUR (2014). Tratado constitutivo de la Unión de Naciones Suramericanas. Secretaría General de UnASUR. Recuperado de File:///D:/ProyecTOS\%2OEN\%2OCURSO/OIM\%2OMMP\%2O20I 5 / UNASUR\%2OTRATADO-UNASUR-SOLO.PDF 